\title{
Characterization and Formation Mechanism of Six Pointed Star-Type Stacking Faults in $4 \mathrm{H}-\mathrm{SiC}$
}

\author{
FANGZHEN WU ${ }^{1}$ HUANHUAN WANG,${ }^{1}$ SHAYAN BYRAPPA, ${ }^{1}$ BALAJI \\ RAGHOTHAMACHAR,${ }^{1}$ MICHAEL DUDLEY $, 1,3$ PING WU, ${ }^{2}$ XUEPING XU, ${ }^{2}$ \\ and ILYA ZWIEBACK ${ }^{2}$ \\ 1.-Materials Science and Engineering, Stony Brook University, Stony Brook, NY, USA. \\ 2.-II-VI Incorporated, Wide Bandgap Materials Group, Pine Brook, NJ, USA. 3.-e-mail: \\ mdudley@notes.cc.sunysb.edu
}

Synchrotron white-beam x-ray topography (SWBXT) studies of defects in 100-mm-diameter $4 \mathrm{H}-\mathrm{SiC}$ wafers grown using physical vapor transport are presented. SWBXT enables nondestructive examination of thick and largediameter $\mathrm{SiC}$ wafers, and defects can be imaged directly. Analysis of the contrast from these defects enables determination of their configuration, which, in turn, provides insight into their possible formation mechanisms. Apart from the usual defects present in the wafers, including micropipes, threading edge dislocations, threading screw dislocations, and basal plane dislocations, a new stacking fault with a peculiar configuration attracts our interest. This fault has the shape of a six-pointed star, comprising faults with three different fault vectors of Shockley type. Transmission and grazing topography of the fault area are carried out, and detailed contrast analysis reveals that the outline of the star is confined by $30^{\circ}$ Shockley partial dislocations. A micropipe, which became the source of dislocations on both the basal plane slip system and the prismatic slip system, is found to be associated with the formation of the star fault. The postulated mechanism involves the reaction of $60^{\circ}$ dislocations of $a / 3\langle\overline{2} 110\rangle$ Burgers vector on basal plane and pure screw dislocations of $a / 3\langle 11 \overline{2} 0\rangle$ Burgers vector on prismatic plane and cross slip of the partial dislocation from prismatic plane to basal plane leading to expansion of the faults.

Key words: Silicon carbide, stacking fault, micropipe, x-ray topography

\section{INTRODUCTION}

The excellent properties of silicon carbide ( $\mathrm{SiC})$ provide a solution to realize the application of power devices under severe conditions such as hightemperature performance, high blocking voltage, and high-frequency switching. ${ }^{1}$ Intense effort is currently in progress to study and eventually eliminate various defects in $\mathrm{SiC}$ in order to improve and stabilize the performance of SiC-based power devices. An active area of research is the origin and expansion of stacking faults in SiC. Although named differently from group to group, three types

(Received August 15, 2012; accepted December 4, 2012; published online January 4, 2013) of stacking faults according to their fault vectors have been reported: Shockley fault with fault vector of $a / 3\langle 1 \overline{1} 00\rangle$ type ${ }^{2-4}$ Frank fault with fault vector of $(c / 2)$ [0001] or (c/4) [0001], ${ }^{5}$ and those comprising some kind of combination of the previous two. ${ }^{6-10}$

Among these faults, Shockley faults have been shown to be associated with the degradation of power devices, as the expansion of such faults in the junction area can impede current flow and, as a result, increase the on-state resistance. ${ }^{3}$ In the epilayer, the morphology of the Shockley faults responsible for the device degradation is found to have a rhombus shape with the sides along $\langle 11 \overline{2} 0\rangle$ directions and angles of $60^{\circ}$ and $120^{\circ}$, bounded by $30^{\circ}$ partial dislocation loops. The fault expands though a mechanism whereby the Si-core partials 
are electrically active, while the C-core partials are not, and the Si-core partials can couple with electron-hole recombination and move.

In $\mathrm{SiC}$, Shockley faults can also form in response to the applied stresses in the crystal. Above the brittle-to-ductile transition temperature (BDT), Shockley faults separate the Si-core and C-core partial dislocations that move in tandem but with separation on the order of $30 \mathrm{~nm}$ to $70 \mathrm{~nm}$. Below the BDT, the C-core partial becomes sessile and thus the Shockley fault can expand as the mobile Sicore partial glides. ${ }^{2}$ Another mechanism of fault expansion in $\mathrm{SiC}$ crystals is related to the existence of threading dislocations with Burgers vectors of $c+a^{4,11,12}$ It is postulated that two partials associated with the $a$-component of the dislocation are separated by a surface step associated with the $c$-component of the deflected $c+a$ dislocation, causing one to be sessile and the other glissile. The Shockley fault created by deflection of a $c+a$ dislocation is observed to be bounded by a straight sessile partial on one side and a curved glissile partial on the other side. The star stacking fault we report herein has a different configuration from those previously studied, and thus a mechanism for its formation is proposed to adapt to its configuration.

\section{EXPERIMENTAL PROCEDURES}

Longitudinally cut, 100-mm-diameter 4H-SiC wafers grown by the physical vapor transport technique were used in our study. SWBXT transmission images of $\{1 \overline{1} 00\},\{\overline{1} 101\}$, and $\{11 \overline{2} 0\}$ reflections were recorded using Agfa Structurix D3-SC film. Grazing-incidence images of the $11 \overline{2} 8$ reflection were recorded from $\mathrm{Si}$-faces of the specimens at x-ray incident angle of $2^{\circ}$. Imaging was carried out at the Stony Brook Synchrotron Topography Station, beamline X-19C, at the National Synchrotron Light Source at Brookhaven National Laboratory.

\section{RESULTS AND DISCUSSION}

\section{Characterization of Six-Pointed Star Stacking Faults}

Figure 1a shows contrast from the stacking fault configuration of interest, which is in the shape of a six-pointed star. The contrast is composed of six rhombuses which share one common corner. It is evident that the contrast of each rhombus is not uniform in that it appears that each large rhombus contains one or more smaller rhombuses inside. This is caused by overlapping of contrast from stacking faults on top of each other in different layers of basal planes which are all projected onto one plane of the film, forming a plan-view image. In other words, each rhombus of the contrast from the star stacking fault is actually from stacking faults on multiple basal planes. The greater the number of overlapping faults, the stronger the contrast would be. It is clear in the image that all faults are connected to the center of the star.

The star stacking fault shows strong contrast in all $\{1 \overline{1} 00\}$ reflections, as in one example shown in Fig. 1a, and also in all $\{\overline{1} 101\}$ reflections, an example of which is shown in Fig. 1b. However, it shows no contrast in all $\{11 \overline{2} 0\}$ reflections, as shown in Fig. 1d-f. According to our previous understanding of the contrast behavior of various stacking faults, ${ }^{4,9,10}$ the fault vector can be determined to be of Shockley type of any of the three $a / 3\langle 1 \overline{1} 00\rangle$ fault vectors.

The three $\{11 \overline{2} 0\}$ reflections also enable determination of the Burgers vectors of the partials bounding the stacking faults, as shown in Fig. 1d-f. Since the stacking faults are out of contrast in these three reflections, the partial dislocations bounding the faults can be clearly observed. However, no reflection of $\langle 11 \overline{2} 0\rangle$ type shows the complete outline of the star stacking fault, and in each reflection the outline of a pair of rhombuses is missing. The Burgers vector of the partials at the edges of the missing rhombuses can be determined through contrast extinction criteria, whereby a dislocation is out of contrast when $g \cdot b$ is equal to zero (where $b$ is the Burgers vector and $g$ is the reflection vector). As a result, the Burgers vector of the partials connected to points 1 and 4 indicated in Fig. 1a is determined to be $(a / 3)$ [1100], as they are out of contrast in the $11 \overline{2} 0$ reflection in Fig. 1d; the Burgers vector of those connected to points 2 and 5 is $(a / 3)$ [0110], as they are out of contrast in the $2 \overline{1} \overline{1} 0$ reflection in Fig. 1e; and the Burgers vector of those connected to points 3 and 6 is $(a / 3)$ [1010], as they are out of contrast in the $1 \overline{2} 10$ reflection in Fig. 1f. It is also noticed that all the partials bounding the star stacking faults lie along $\langle 11 \overline{2} 0\rangle$ directions and make angles of $30^{\circ}$ to their Burgers vectors.

The same area containing the star stacking fault was studied using grazing-incidence topography, as shown in Fig. 1c. Contrast from a small micropipe is found in the center of the star stacking fault. It can also be seen that four linear bands of dislocations emanate from the micropipe, while the two linear bands of dislocations which are parallel to the reflection vector direction indicated in the image are out of contrast. None of the six bands of dislocations emanating from the micropipe ever completely disappear in any of the $\{11 \overline{2} 0\}$ or $\{1 \overline{1} 00\}$ reflections, which implies that none of the bands are composed of dislocations with a single Burgers vector. Instead, each band of dislocations should include dislocations with at least two different Burgers vectors of any $(a / 3)\{11 \overline{2} 0\}$ type or $(a / 3)\{1 \overline{1} 00\}$ type.

At this point, the configuration of star stacking faults can be summarized to be the shape of a sixpointed star comprising faults with three different fault vectors of Shockley type. The outline of the star is confined by $30^{\circ}$ Shockley partial dislocations lying along $\langle 11 \overline{2} 0\rangle$ directions. A micropipe is located in the center of the star stacking fault, from which 

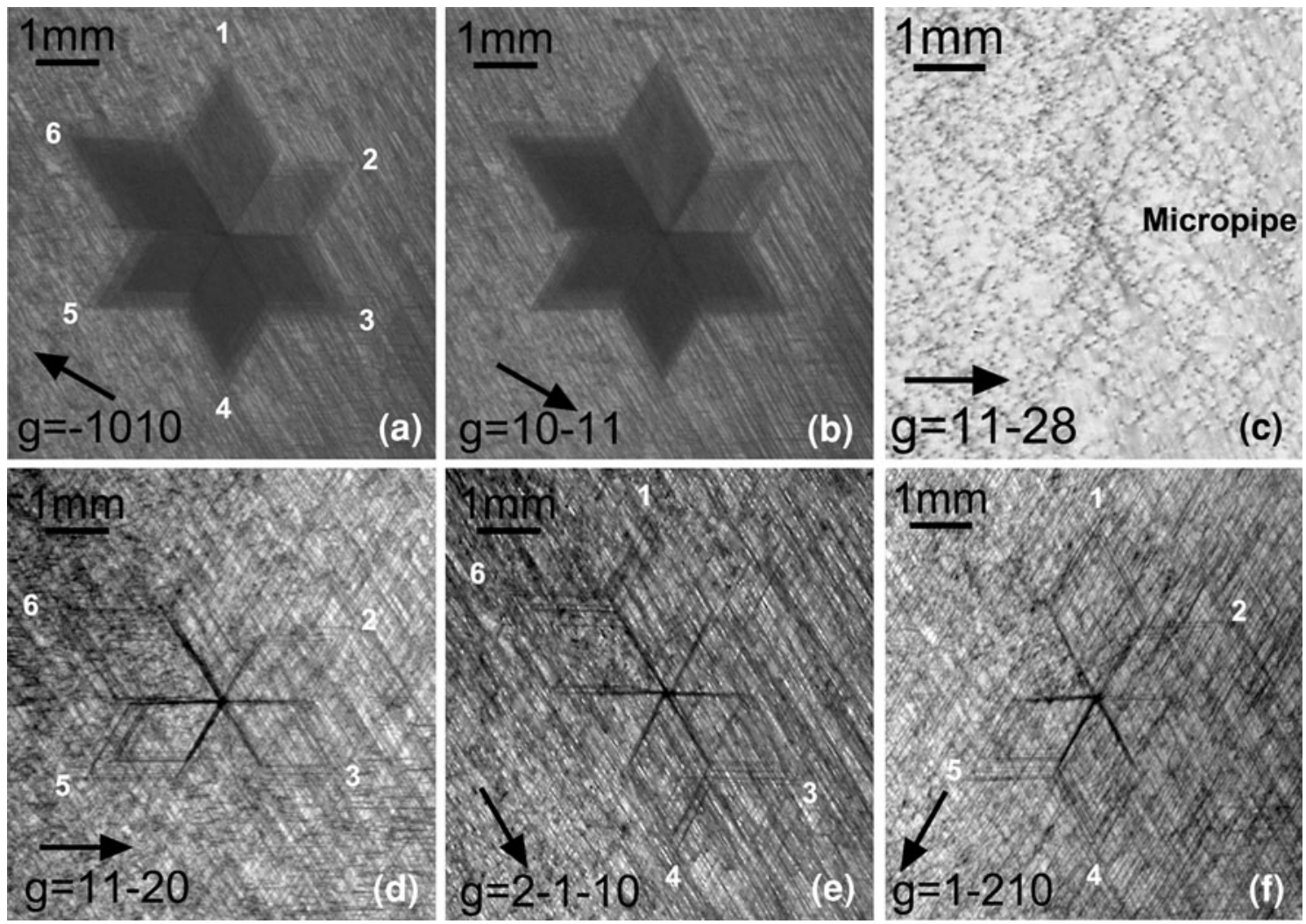

Fig. 1. SWBXT images recorded from a region in a $4 \mathrm{H}-\mathrm{SiC}$ crystal containing six-pointed star stacking faults: (a) transmission image of 1010 reflection where each point of the star is labeled from 1 to 6 ; (b) transmission image of $10 \overline{1} 1$ reflection; (c) grazing-incidence image of $11 \overline{2} 8$ reflection, revealing that a small micropipe is located in the center of the star stacking fault; (d-f) transmission images of three $\{1120\}$ reflections showing the absence of the fault contrast as well as partial dislocations connected to those missing points in each image.

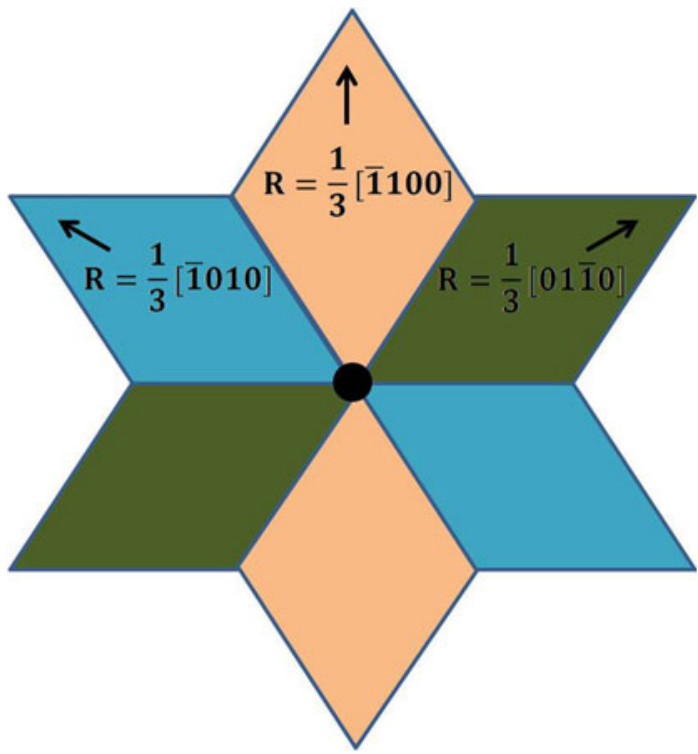

Fig. 2. Schematic of the configuration of six-pointed star stacking faults.

emanate six bands of dislocations with a mixture of Burgers vectors. A schematic representation of the configuration is shown in Fig. 2.

\section{Formation Mechanism of Six-Pointed Star Stacking Faults}

The background of the star stacking fault in Fig. 1a comprises straight basal plane dislocations (BPDs) whose contrast is displayed as black lines. Despite their large number in Fig. 1a, it can be identified that they are mainly oriented toward [2 110$]$ and [11 $\overline{2} 0]$ directions. Two more $\{1 \overline{1} 00\}$ reflections recorded from the same area are shown in Fig. 3. In the background of Fig. 3a, BPDs are oriented toward [1210] and $[11 \overline{2} 0]$ direction, but BPDs along $[\overline{2} 110]$ direction all disappear in $0 \overline{1} 10$ reflection. In the background of Fig. $3 b, B P D$ are oriented toward [ $\overline{2} 110]$ and [12 10$]$ directions, but BPDs along [1120] direction all disappear in (1100) reflection. Therefore, by applying $g \cdot b$ analysis, the Burgers vectors of the missing BPDs in each reflection can be determined, and it turns out that they are all pure screw dislocations with Burgers vector of $(a / 3)\langle 11 \overline{2} 0\rangle$. These pure screw dislocations are the precursor to the mechanism of formation of the star stacking fault configuration.

The micropipe located in the center of the star stacking fault is another major player in the formation mechanism. It is known that micropipes can be the source of BPDs. ${ }^{13}$ The BPDs generated from a micropipe in basal plane slip system $\langle 11 \overline{2} 0\rangle(0001)$ 

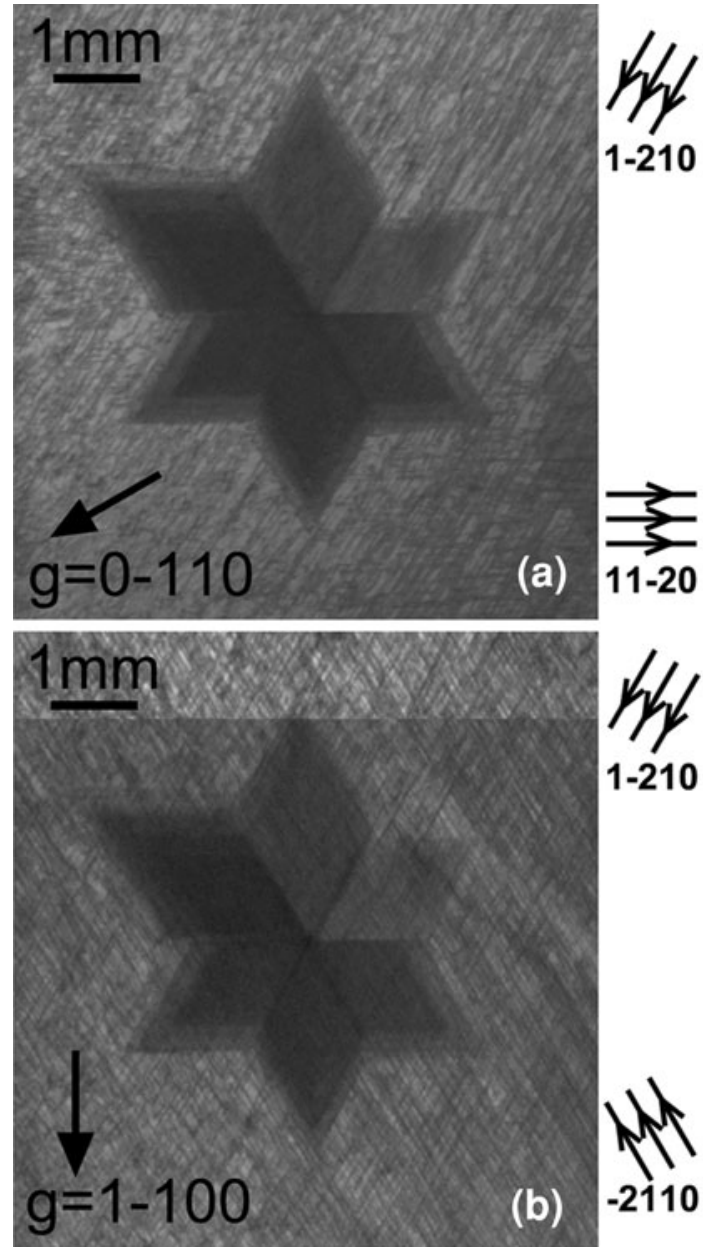

Fig. 3. SWBXT images recorded from the region containing a sixpointed star stacking fault: (a) transmission image of $0 \overline{1} 10$ reflection; (b) transmission image of $1 \overline{100}$ reflection.

can have different character, i.e., different angles between their Burgers vectors and line directions. It is well known that the mobility of non-screw dislocations is higher than that of screw dislocations in $\mathrm{SiC},{ }^{2}$ and thus non-screw BPDs move more easily in response to stress above a certain temperature. Therefore, non-screw dislocations generated from the micropipe are seldom observed in the background of the images of our area of interest. In addition, dislocations generated from micropipes in the prismatic slip system $\langle 11 \overline{2} 0\rangle\{1 \overline{1} 00\}$ have also been reported. ${ }^{14,15}$ Furthermore, screw character dislocations with Burgers vectors of $(a / 3)\langle 11 \overline{2} 0\rangle$ can cross slip from prismatic plane $\{1 \overline{1} 00\}$ onto basal plane. ${ }^{16}$

In our model for the formation mechanism of the star stacking fault configuration, dislocations with Burgers vector of $(a / 3)\langle 11 \overline{2} 0\rangle$ are nucleated from a micropipe in both the basal plane slip system and prismatic slip system, as shown in Fig. 4a. The dislocations which are generated in the prismatic slip system which have screw character can later cross slip onto the basal plane. The dislocations

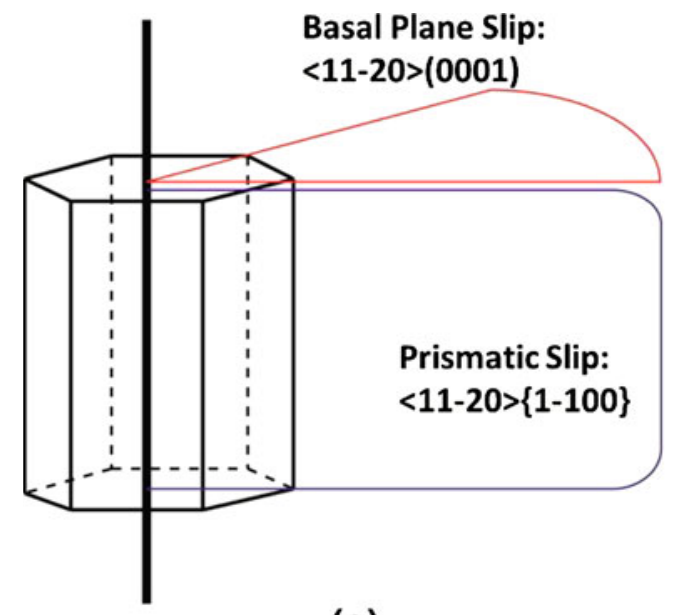

(a)

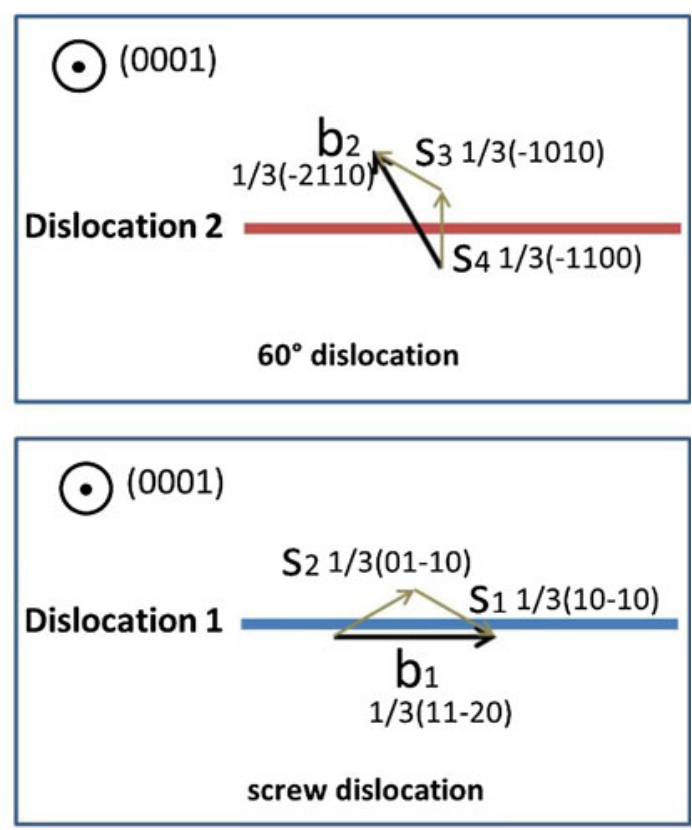

(b)

Fig. 4. (a) Schematic drawing of dislocations nucleating from a micropipe in both the basal plane slip system and prismatic slip system; (b) Burgers vectors and partials of a $60^{\circ}$ dislocation and a screw dislocation on basal plane.

which are generated in basal plane which are operative in our model have $60^{\circ}$ character (i.e., $60^{\circ}$ between their Burgers vector and line direction). One example of these two types of dislocations as well as their partials is illustrated in Fig. 4b. Dislocation 1 is generated in prismatic slip system but cross slips into basal plane. It has to be of pure screw type, and S1 and S2 are its partials. Dislocation 2 is appointed to be generated in basal plane slip system and is a $60^{\circ}$ dislocation whose partials are S3 and S4. Dislocations 2 and 1 may or may not be in different basal planes at different levels. It should be noted that the Burgers vectors of S3 and S1 are of opposite sign. Strong attractive forces 
might therefore be expected between these two partials.

The force between these dislocations is calculated in order to understand their mutual influence. According to dislocation theory, ${ }^{17}$ the total force $F$ exerted on a unit length of a mixed dislocation from another mixed dislocation is

$$
F=-\left(\sigma_{x y} b_{x}+\sigma_{y z} b_{z}\right) i+\left(\sigma_{x x} b_{x}+\sigma_{x z} b_{z}\right) j
$$

where $b_{x}$ and $b_{z}$ are components of the Burgers vector of the former dislocation and $\sigma_{x x}, \sigma_{x y}, \sigma_{y z}$, and $\sigma_{x z}$ are components of the stress field arising from the latter dislocation. The origin of the coordinate system is set at the dislocation line; the $z$-axis is parallel to the dislocation line direction; the $x$-axis is perpendicular to the dislocation line and lies in the slip plane; the $y$-axis is perpendicular to the slip plane.

For the case of our model, where the dislocations are parallel to each other and may lie in separate basal planes, only the first component of $F$ in Eq. 1 needs to be calculated, as it is responsible for the glide of the dislocation on the basal plane.

For a screw dislocation,

$$
\sigma_{y z}=-\frac{\mu b}{2 \pi} \cdot \frac{x}{x^{2}+y^{2}} ; \sigma_{x y}=0
$$

For an edge dislocation,

$$
\sigma_{x y}=-\frac{\mu b}{2 \pi(1-v)} \cdot \frac{x\left(x^{2}-y^{2}\right)}{\left(x^{2}+y^{2}\right)^{2}} ; \sigma_{y z}=0,
$$

where $b$ is the Burgers vector of the dislocation, $\mu$ is the shear modulus, and $v$ is the Poisson's ratio. A mixed dislocation can be regarded as the combination of a screw and an edge dislocation.

Therefore, the force exerted on S1 from dislocation 2 is

$$
-\frac{3 s^{2} t \mu}{8 \pi\left(h^{2}+t^{2}\right)}-\frac{3 s^{2} t\left(-h^{2}+t^{2}\right) \mu}{8 \pi\left(h^{2}+t^{2}\right)^{2}(1-v)},
$$

and the force exerted on $\mathrm{S} 2$ from dislocation 2 is

$$
-\frac{3 s^{2} t \mu}{8 \pi\left(h^{2}+t^{2}\right)}+\frac{3 s^{2} t\left(-h^{2}+t^{2}\right) \mu}{8 \pi\left(h^{2}+t^{2}\right)^{2}(1-v)},
$$

where $s$ represents the magnitude of the partial's Burgers vector, $h$ is the separation of two basal planes upon which dislocations 1 and 2 lie, and $t$ is the distance between two dislocations projected onto the basal plane. Two situations are considered. The first situation is when dislocations 1 and 2 are on the same basal plane, which means $h=0$, though the chance of this situation might be relatively small. Taking $v=0.2$, Eq. 4 becomes $-\frac{27 s^{2} \mu}{32 \pi t}$ and Eq. 2 becomes $\frac{3 s^{2} \mu}{32 \pi t}$. This can be interpreted as dislocation 2 having an attractive force on $\mathrm{S} 1$ and a repulsive force on $\mathrm{S} 2$.
In other words, $\mathrm{S} 1$ becomes sessile and $\mathrm{S} 2$ becomes glissile, and under certain stress $\mathrm{S} 2$ can start to move, leaving a Shockley fault behind in its wake.

The second situation is when dislocations 1 and 2 are on two different basal planes. The forces on S1 and S2 exerted from dislocation 2 are plotted in Fig. 5a and c, respectively. These graphs are plotted for a $h$ value of $10 \mathrm{~nm}$, about 10 atomic layers of $4 \mathrm{H}$ $\mathrm{SiC}$ structure. Over a long range of $t$, the forces on $\mathrm{S} 1$ and S2 are opposite, with attractive forces on S1 and repulsive forces on $\mathrm{S} 2$ being apparent, as shown in Fig. 5a, c. A significant difference exists from the first situation when $t$ is very small, that is, when the partial is very close to dislocation 2 along the slip direction. Figure 5b shows the region near the origin of Fig. 5a at much higher magnification. It indicates that $\mathrm{S} 1$ is locked inside a region about $+5 \mathrm{~nm}$ and $-5 \mathrm{~nm}$ from the position right below (or above) dislocation 2 and can no longer glide. As indicated in Fig. 5d, S2 is stable (locked in position) near the origin, but beyond the position of $+30 \mathrm{~nm}$ or $-30 \mathrm{~nm}$ it will experience a continuous repulsive force. Therefore, when dislocation 1 is not nucleated right below (or above) dislocation 2 but with enough mutual lateral shift, the two partials will separate as soon as dislocation 1 cross slips from the prismatic plane to the basal plane and one partial becomes sessile and the other becomes glissile.

In the same way, one can also calculate the force on S3 and S4 exerted from dislocation 1. The force on S3 is negative, as expected due to the opposite sign of its Burgers vector with respect to S2. The force on S4 turns out to be zero. Then, it becomes obvious that $\mathrm{S} 2$ is most likely to glide in response to stress and lead to expansion of a Shockley fault, since it is the only one among the four partials that can receive continuous repulsive force over a long range.

As $4 \mathrm{H}-\mathrm{SiC}$ is sixfold symmetric along (0001) growth direction, the dislocation reaction discussed above can happen between all dislocations nucleated at the micropipe core on the six symmetryrelated prismatic planes and dislocations generated on the basal plane. Those screw segments of dislocations generated on the prismatic plane can cross slip onto the basal plane, and the glissile partials, which are $30^{\circ}$ dislocations, start to move in response to stress, leaving Shockley faults in their wake. The six pointed star-shaped stacking fault configuration forms as the glide of Shockley partials occurs respectively in all six slip directions. Figure 6 illustrates a moment when the star pattern of such a stacking fault is forming. The solid lines represent the position of partials bounding the Shockley faults already formed, and the dashed lines represent the final positions of partials bounding the star stacking fault configuration. The image shows the situation when an $(a / 3)$ [0110] Shockley fault is expanding toward the direction indicated by the black arrows, while fault $(a / 3)$ [1100] is already formed and fault $(a / 3)$ [1010] has not yet formed. Eventually, the 


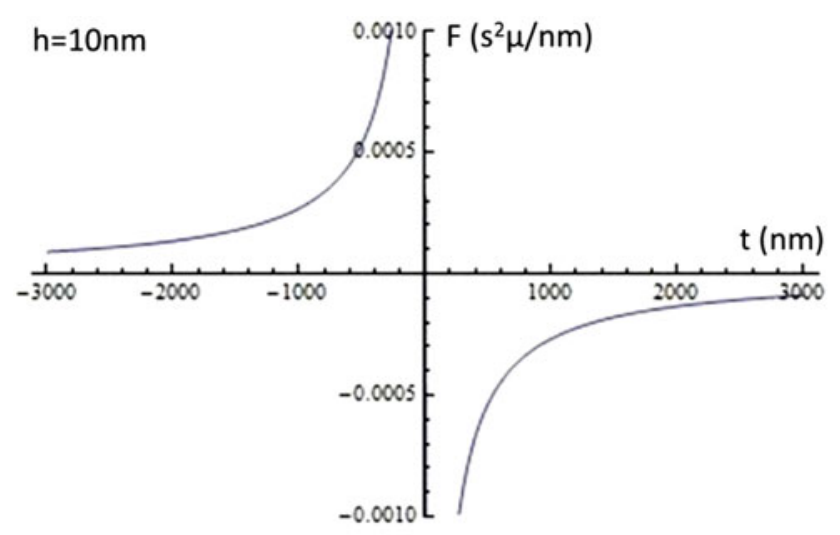

(a)

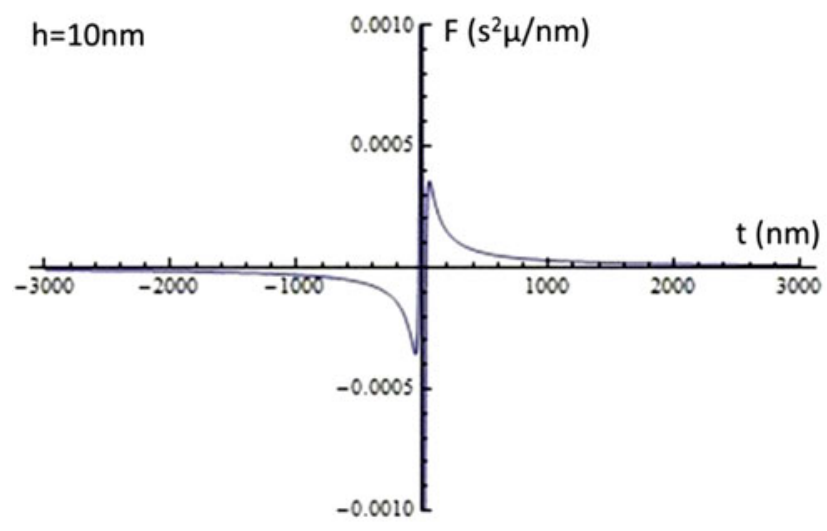

(c)

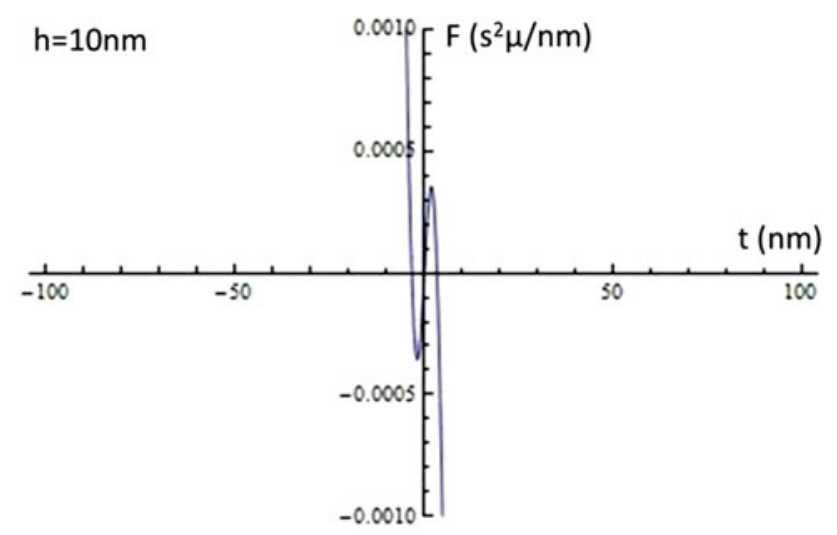

(b)

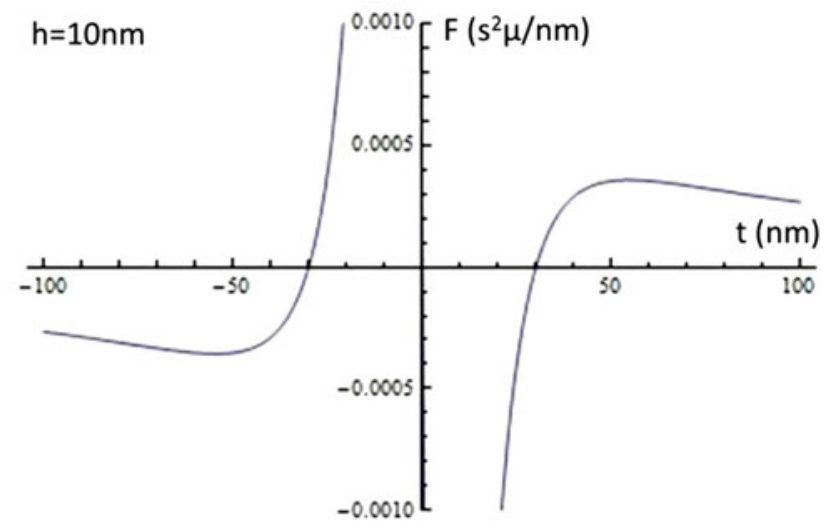

(d)

Fig. 5. The forces on $\mathrm{S} 1$ and $\mathrm{S} 2$ exerted by dislocation 2: (a) the force on $\mathrm{S} 1, t$ in range [-3000, 3000]; (b) the force on $\mathrm{S} 1, t$ in range [-100, 100]; (c) the force on S2, $t$ in range [-3000, 3000]; (d) the force on S2, $t$ in range $[-100,100]$. The unit of force on dislocation line, $F$, is $\left(s^{2} \mu / n_{m}\right)$. Diagrams are plotted with software Mathematica 8.

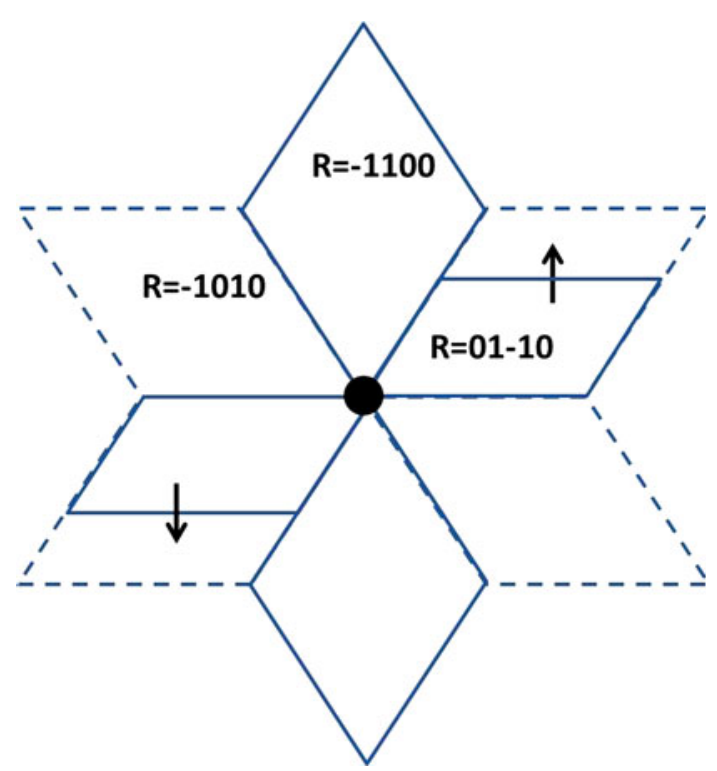

Fig. 6. Illustration of a snapshot when a six-pointed star shaped stacking fault is forming. complete star stacking fault configuration will be like the one illustrated in Fig. 2.

\section{CONCLUSIONS}

Stacking faults in a configuration shaped like a six-pointed star comprise faults with three different fault vectors of Shockley type. The outline of the star is confined by $30^{\circ}$ Shockley partial dislocations lying along $\langle 11 \overline{2} 0\rangle$ directions. A micropipe is located in the center of the star stacking fault, from which emanate six bands of dislocations with a mixture of Burgers vectors. It is postulated that $60^{\circ}$ dislocations of $a / 3\langle\overline{2} 110\rangle$ Burgers vector on basal plane and pure screw dislocations of $a / 3$ $\langle 11 \overline{2} 0\rangle$ Burgers vector on prismatic plane nucleate from the micropipe. The screw dislocations can cross slip from prismatic plane to basal plane. Then, two partials of a screw dislocation start to separate under stress because one partial is sessile and the other one glissile due to the mutual influence of the force from dislocations generated in the two different slip systems. Shockley faults 
form and expand as a result of glide of the glissile partials. Six-pointed star stacking faults appear when the glide of Shockley partials occurs in all six slip directions that are perpendicular to the dislocation line direction.

\section{ACKNOWLEDGEMENTS}

This work was supported by II-VI Incorporated. Topography experiments were carried out at the Stony Brook Synchrotron Topography Facility, beamline X-19C, at the NSLS, BNL (DOE Office of Basic Energy Sciences Contract No. DE-AC02-76 CH00016).

\section{REFERENCES}

1. R. Singh and M. Pecht, IEE Ind Electron. Mag. 2, 19 (2008).

2. P. Pirouz, J.L. Demenet, and M.H. Hong, Philos. Mag. A 81, 1207 (2001).

3. M. Skowronski and S. Ha, J. Appl. Phys. 99, 011101 (2006).

4. M. Dudley, S. Byrappa, H. Wang, F. Wu, Y. Zhang, B. Raghothamachar, G. Choi, E.K. Sanchez, D. Hansen, R. Drachev, and M.J. Loboda, Mater. Res. Soc. Symp. Proc. 1246, B02-02 (2010).

5. H. Tsuchida, I. Kamata, and M. Nagano, J. Cryst. Growth 310, 757 (2008).
6. M. Benamara, X. Zhang, M. Skowronski, P. Ruterana, G. Nouet, J.J. Sumakeris, M.J. Paisley, and M.J. O’Loughlin, Appl. Phys. Lett. 86, 021905 (2005).

7. H. Chen, G. Wang, Y. Chen, X. Jia, J. Bai, and M. Dudley, Mater. Res. Soc. Symp. Proc. 911, 0911-B05-24 (2006).

8. H. Tsuchida, I. Kamata, and M. Nagano, J. Cryst. Growth 306, 254 (2007).

9. M. Dudley, H. Wang, F. Wu, S. Byrappa, B. Raghothamachar, G. Choi, E.K. Sanchez, D. Hansen, R. Drachev, S.G. Mueller, and M.J. Loboda, Mater. Sci. Forum 679-680, 269 (2011).

10. M. Dudley, F. Wu, H. Wang, S. Byrappa, B. Raghothamachar, G. Choi, E.K. Sanchez, D. Hansen, R. Drachev, S.G. Mueller, and M.J. Loboda, Appl. Phys. Lett. 98, 232110 (2011).

11. F. Wu, H. Wang, S. Byrappa, B. Raghothamachar, M. Dudley, E.K. Sanchez, D. Hansen, R. Drachev, S.G. Mueller, and M.J. Loboda, Mater. Sci. Forum 717-720, 343 (2012)

12. F. Wu, S. Byrappa, H. Wang, Y. Chen, B. Raghothamachar, M. Dudley, E. K. Sanchez, G. Chung, D. Hansen, S. G. Mueller, and M. J. Loboda, Mater. Res. Soc. Symp. Proc. 1433 (2012).

13. M. Dudley, X. Huang, and W.M. Vetter, J. Phys. D Appl. Phys. 36, A30 (2003).

14. S. Ha, N.T. Nuhfer, G.S. Rohrer, M. De Graef, and M. Skowronski, J. Electron. Mater. 29, L5 (2000).

15. H. Wang, S. Byrappa, F. Wu, B. Raghothamachar, M. Dudley, E.K. Sanchez, D. Hansen, R. Drachev, S.G. Mueller, and M.J. Loboda, Mater. Sci. Forum 717-720, 327 (2012).

16. A. Mussi, J.L. Demenet, and J. Rabier, Phil. Mag. Lett. 86 , 561 (2006)

17. J. Weertman and J.R. Weertman, Elementary Dislocation Theory (USA: Oxford University Press, 1992), p. 58. 